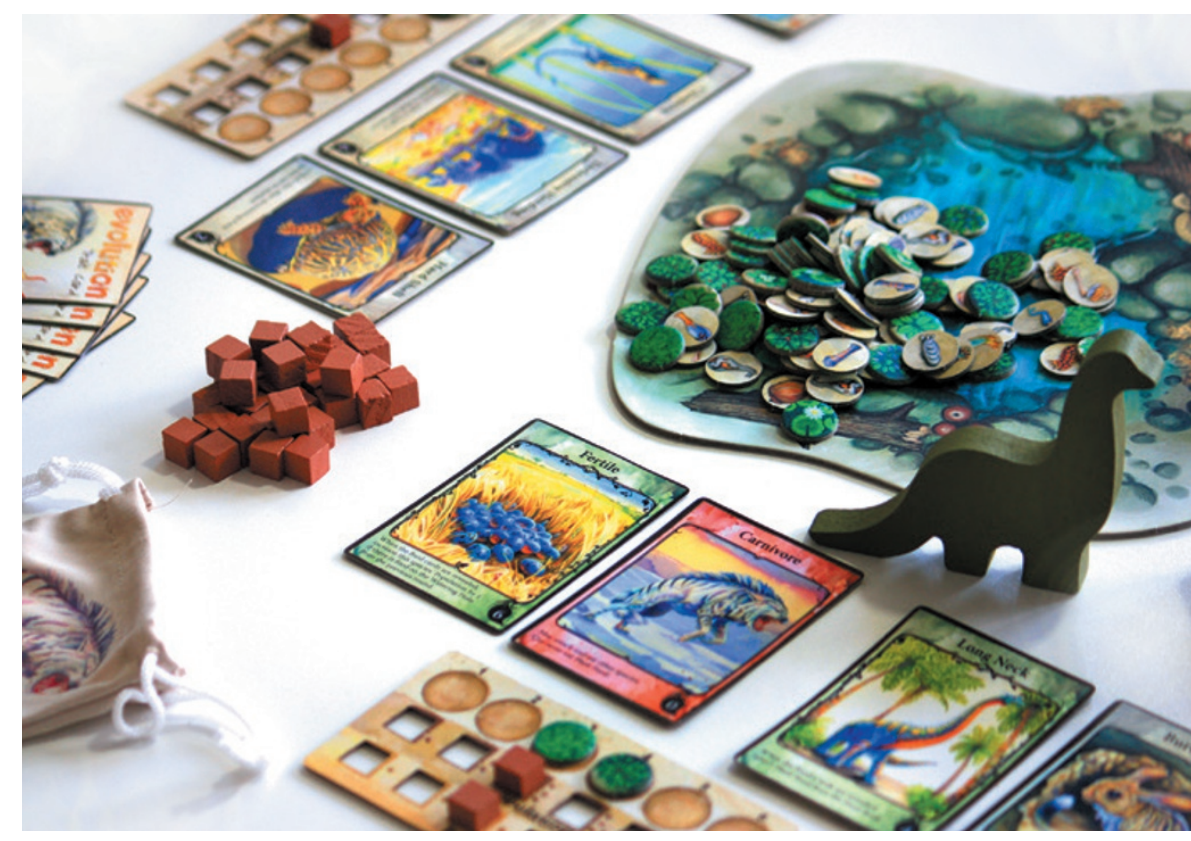

In Evolution, players use cards to develop species — and win points for their survival.

\section{How to win at evolution}

\section{Stuart West and helpers compare the cut and thrust of three games that explore life's greatest competition.}

volution by natural selection is like a game: the winners are the organisms best at passing on their genes. Several board games are now available that exploit the strategies and uncertainties involved. We (two 12 year olds, a 16 year old, three graduate students, two postdocs and three professors) tested three: Evolution by North Star Games, Evolution: Random Mutations by Rightgames and Terra Evolution: Tree of Life by Mindwarrior Games. Our mission? To discover their potential for 'edutainment' — and as last-minute holiday gifts.

Evolution focuses on adaptation and interspecies interactions. You fashion your species, which competes for a food supply. Players are dealt cards allowing them to start new herbivore species, give an existing species a bigger population or bigger body, or add an advantage such as improved efficiency at foraging or the ability to store food as fat. Some cards turn a herbivore into a carnivore. Some help herbivores to avoid carnivores, with a shell or warning calls; other attributes, such as pack hunting and intelligence, help predators to evade these defences. Efficient herbivores get more food, but can be eaten by carnivores. Points are scored for eating, and for the species you have left at the end of the game. The player with the most points wins.

The same focus and similar mechanics
Evolution

North Star Games: 2014.

Terra Evolution: Tree of Life

Mindwarrior Games: 2015.

Evolution: Random Mutations

Rightgames: 2013.

pervade Evolution: Random Mutations, but you are not allowed to know the ability on a card before you add it to a species. The game also has cards that derail a species' survival, such as developmental defects. Points accrue according to the number and complexity of the species you have at the end of the game, along with traits such as high body weight, which make a species need more food.

The more complex Terra Evolution offers a nice illustration of how the tree of life is built over time, and opens discussions about the repeatability of evolution. You start with seven continents on a planet and a simple, worm-like animal. You build a deck of cards allowing you to evolve complex organisms, fossilize others or attack rival players with catastrophes such as viruses or asteroids. Complexity in an animal can be a step towards greater complexity: fish lead to reptiles, and reptiles to birds or mammals. Or a complex animal might be an evolutionary dead-end, but give you a useful trait wasps, for example, help you to attack other players. The winner is the first to evolve both mammals and birds, and to score a certain number of fossil points.

We savoured all three games, but Evolution is our favourite by far. It looks amazing, with evocative artistry in everything from the cards to the little animal drumsticks that the predators eat. A gratuitous wooden dinosaur marks whose turn it is, and tactile, satiny bags store eaten food. The gameplay is simple to grasp, but can get very tactical. In particular, as with real evolution, the best strategy depends on what everyone else is doing. If there are a lot of herbivores, there is an advantage to being an efficient forager, with traits such as cooperation, but lots of herbivores also means a big advantage to becoming a carnivore. When carnivores appear, herbivores need defences, which carnivores try to get around - and so on, in a co-evolutionary dance.

Evolution features sophisticated biology. Traits can be put together in a dizzying array of combinations, so each game can be very different. The theme of evolution is not just tacked on: it drives play. A long neck gets you food that would not normally be available. Symbiosis means that you can only be eaten by a predator if it eats your host first. Horns do not stop you being eaten, but they damage predators, and so act as a deterrent.

Evolution captures key aspects of the evolutionary process and would work as a teaching aid for ages ten and up. It could also help older students to tackle specific topics, such as evolutionary arms races. Random Mutations is neither as attractive nor as fun, the welcome addition of parasites notwithstanding, but its small size could make it a good travel game. It is a little drier, although it does a great job of illustrating the random role of mutation, which can increase or decrease fitness.

Terra Evolution was the hardest to grasp we had to play several times to get the hang of it. How you play early on can have long-term consequences, and the best strategy can vary with the stage of the game. We liked the ability to wreak cataclysms, although this worked best with fewer players. It would suit older players, who like more complex gameplay.

All the games, and especially Evolution, deftly capture how natural selection produces organisms adapted to their environments. I would love to see a board game exploring the dynamics of natural selection within species, through changes in gene frequency. But making population genetics fun is no mean feat. In the meantime, I am delighted to see so many attempts to drum up understanding of this greatest of all games.

Stuart West is Professor of Evolutionary Biology in the Department of Zoology at the University of Oxford, UK. He studies adaptation in organisms ranging from bacteria to humans.

e-mail:stuart.west@zoo.ox.ac.uk 